\title{
Implementación de Aplicaciones Informáticas en la Industria Agrícola del Aguacate
}

\author{
Implementation of Computer Applications in the Avocado Agriculture Industry
}

OCHOA-ORNELAS, Raquel $\dagger^{*}$, FAJARDO-DELGADO, Daniel, SÁNCHEZ-CERVANTES, María Guadalupe y OSORNIO-MENDOZA, Jonathan

Instituto Tecnológico de Ciudad Guzmán / Tecnológico Nacional de México

ID $1^{\text {er }}$ Autor: Raquel, Ochoa-Ornelas / ORC ID: 0000-003-1824-5789, Researcher ID Thomson: S-4687-2018, arXiv Author ID: RaquelOchoa, CVU CONACYT ID: 668976

ID $1{ }^{\text {er }}$ Coautor: Daniel, Fajardo-Delgado / ORC ID: 0000-0001-8215-5927, Researcher ID Thomson: B-5078-2014, arXiv Author ID: dfajardod, CVU CONACYT ID: 101301

ID $2^{\mathrm{do}}$ Coautor: María Guadalupe, Sánchez-Cervantes / ORC ID: 0000-0002-5517-9816, Researcher ID Thomson: S- 67842018, arXiv Author ID: magusace, CVU CONACYT ID: 544127

ID $3^{\text {er }}$ Coautor: Jonathan, Osornio-Mendoza / ORC ID: 0000-0002-5310-0369, Researcher ID Thomson: S- 5751-2018, arXiv Author ID: john9651, CVU CONACYT ID: 946995

DOI: $10.35429 / J T D .2019 .9 .3 .13 .23$

Recibido: 10 de Enero, 2019; Aceptado 30 de Marzo, 2019

\section{Resumen}

La Agricultura 4.0, concebida como una especie de industria, se construye mediante la integración de un conjunto de tecnologías digitales tales como el Internet de las cosas, la Inteligencia Artificial, y la gestión de grandes bases de datos (o Big Data). Esto implica una transformación de la infraestructura de producción que permite incrementar la productividad agrícola y la calidad de los productos de una manera eficiente y sostenible. Estos cambios requieren de la implementación de tecnologías digitales para la recolección, el análisis y la comunicación de información, permitiendo la toma de decisiones oportuna. El presente artículo trata de la implementación de una plataforma que comprende tanto aplicaciones de escritorio, Web y móviles como herramientas de Internet de las cosas para el control de la producción y la comercialización en huertas aguacateras. Se describe el desarrollo de un prototipo de la plataforma bajo metodologías ágiles y siguiendo una estrategia iterativa e incremental. Finalmente, se presentan las pruebas que determinan la funcionalidad integral del prototipo de la plataforma y resultados experimentales.

Agricultura 4.0, Conectividad, Base de Datos

\begin{abstract}
Agriculture 4.0, conceived as a kind of industry, is built by integrating a set of digital technologies such as the Internet of things, Artificial Intelligence, and the management of large databases (or Big Data). This industry implies a transformation of the production infrastructure that allows increasing the agricultural productivity and the quality of the products efficiently and sustainably. These changes require the implementation of digital technologies for the collection, analysis and communication of information, allowing timely decision making. This article deals with the implementation of a platform that includes both desktop, Web and mobile applications and Internet of things tools for the control of production and marketing in avocado orchards. The development of a prototype of the platform is described under agile methodologies and following an iterative and incremental strategy. Finally, the tests that determine the integral functionality of the platform prototype and experimental results are presented.
\end{abstract}

Agriculture 4.0, Connectivity, Database

Citación: OCHOA-ORNELAS, Raquel, FAJARDO-DELGADO, Daniel, SÁNCHEZ-CERVANTES, María Guadalupe y OSORNIO-MENDOZA, Jonathan. Implementación de Aplicaciones Informáticas en la Industria Agrícola del Aguacate. Revista del Desarrollo Tecnológico. 2019. 3-9: 13-23

\footnotetext{
* Correspondencia del Autor (Correo electrónico: raqueoo@itcg.edu,mx)

$\dagger$ Investigador contribuyendo como primer autor.
} 


\section{Introducción}

En los últimos años, se ha incrementado considerablemente la producción de las huertas aguacateras en los estados de Jalisco y Michoacán, generando una alta producción que es distribuida a diferentes empacadoras de la región para su posterior exportación. La importancia económica de esta actividad en cuanto a la generación de empleos y los vínculos indirectos con otras actividades es significativa en Jalisco, ya que se han exportado en promedio más de 514,000 toneladas en los últimos años solamente a Estados Unidos de América (Faostat, 2016).

A pesar de los esfuerzos de estas organizaciones por apoyar los procesos productivos, en la actualidad, existen huertas de aguacate en Jalisco y Michoacán que llevan el control de los procesos de forma manual, generando grandes cantidades de papeleo y errores, incrementando el extravío de datos e inconsistencias en la información que afectan el seguimiento al mantenimiento en las huertas y a su producción.

En este artículo, se presenta una plataforma que comprende aplicaciones de escritorio, Web y móviles, así como herramientas de Internet de las cosas para el control de la producción y la comercialización en huertas aguacateras. Las aplicaciones permiten el procesamiento de datos relacionados con las actividades productivas y administrativas de los huertos, almacenando registros en una base de datos instalada en un servidor en la nube, que ofrece además, diversas consultas a través de dispositivos móviles.

\section{Antecedentes}

Desde la primera revolución industrial que se presenta en el siglo XVIII con la aparición de la máquina de vapor, se vio involucrada la agricultura con estos avances, pues existen antecedentes como fue el país de Chile en el siglo XIX, que siendo el principal productor agrícola de la Costa del Pacífico, fue afectado en la reducción de mano de obra en el campo debido a conflictos bélicos, por lo cual tuvo que emplear maquinaria como trilladoras de vapor y segadoras mecánicas en el año de 1880 (Sater, 2019).
Hacia finales del siglo XIX surge la segunda revolución industrial, la cual se basa en el uso de la electricidad para fines industriales y domésticos. En lo que respecta a la Agricultura 2.0 se implementaron soluciones con la electricidad que permitieron incrementar la productividad a través de molinos de aceite. La tercera revolución industrial se presenta a finales del siglo XX involucrando la robotización y la automatización. En esta época, surge la Agricultura 3.0 con el uso de grandes maquinarias que realizan ciclos completos de trabajo tanto en la siembra como en la cosecha (Zarazaga, 2017).

Actualmente, la cuarta revolución industrial se refleja en la agricultura con las explotaciones inteligentes que comprenden sistemas de emplazamiento para la rotación de cultivos, así como de controles que optimizan el riego y fertilizantes entre otros. Sin embargo, existen retos a alcanzar en la agricultura en el entorno industrial actual, como lo es el uso de sensores en las explotaciones por problemas de alimentación energética, así como la transferencia de datos en comunidades sin cobertura de Internet. Los satélites y los drones juegan un papel importante al obtener datos impulsando el desarrollo de la agricultura, pero requieren de sistemas robustos con grandes bases de datos que ofrezcan servicios de consumo masivo. Otro problema más a considerar es la resistencia de los agricultores a incorporar a las nuevas tecnologías en el campo. (Zarazaga, 2017).

Por otra parte, algunos datos generales de la agricultura en América Latina muestran que existe tendencia al desarrollo capitalista ya que se han expropiado las mejores tierras en extensiones medias y grandes en varios países, cuyas inversiones extranjeras o privadas encuentran beneficios directos en infraestructura e incentivos económicos. Sin embargo, los pequeños campesinos carecen de apoyos $\mathrm{y}$ utilizan técnicas atrasadas y poco especializadas donde su producción se encuentra sujeta al clima o tradiciones agrícolas, quedando limitados al uso de la tecnología (Pérez y Reyes, 2019).

Bernal et al. (2019) definen al seguro agrícola como un soporte financiero en riesgos a eventos climáticos adversos que no se pueden prevenir de manera anticipada. 
En Bolivia, el seguro agrario universal creado en 2011 que responde de manera oportuna para albergar sistemas de producción, comercio y seguro para la agricultura familiar, alberga a más de 100,000 productores agrícolas. En algunos países de América Latina el crédito a los pequeños productores se ve limitado precisamente por no disponer de este tipo de seguros, garantías o la falta de títulos de propiedad. Lo que hace una desigualdad más grande en unidades de producción, nivel tecnológico e ingresos. Una solución a todos estos problemas es otorgando el apoyo al campo a través de la asistencia y capacitación en el uso de nueva tecnología, administración, comercialización, así como la vinculación con los mercados (Pérez y Reyes, 2019).

Calvo (2019) expone que las Tecnologías de la Información y la Comunicación (TIC) son consideradas un pilar importante en la economía y sociedad actual ya que son de uso general y su aplicación es fundamental para el crecimiento y desarrollo. Por tal motivo, explica el caso de la empresa INDRA que combina capital público y privado del sector TIC en España, misma que se han consolidado en los últimos años al centrarse en ajustar el desarrollo tecnológico potenciando la ingeniería de sistemas, optimizando tecnologías y participando en programas nacionales e internaciones de innovación y desarrollo, recibiendo apoyos de diferentes organismos incluyendo la Unión Europea y el Ministerio de Industria y Energía.

Pérez-Garcia (2019) propone un sistema de información geográfico en la agricultura cañera para la gestión de parcelas. La plataforma está integrada por un software de gestión, un sistema gestor de base de datos y una interfaz Web, que involucran información espacial de áreas de cultivo de caña de azúcar y datos de cosecha. Esta plataforma mejora significativamente la gestión y el control de los insumos empleados durante las tareas habituales aumentando la eficiencia. Además, a partir de las imágenes aéreas y otras herramientas se obtienen imágenes satelitales multiespectrales que producen mapas de prescripción permitiendo efectuar un tratamiento diferenciado del cultivo en función de las necesidades de los mismos. Por otra parte, a través de la plataforma se generan otros indicadores de productividad que aportan soluciones a decisiones administrativas $y$ planeación futura.
Méndez-Moreno et al. (2019) declaran que los códigos QR (Quick Response, por sus siglas en inglés), permiten acceder de manera rápida a la información, ya que al ser bidireccional, puede ser leído mediante la cámara de un dispositivo móvil y una aplicación lectora. La investigación describe la adquisición de requerimientos para la implementación de tecnología QR que permita acceder a información de las orquídeas del museo viviente de las orquídeas en el estado de Yucatán. Mencionan además, que la agricultura requiere actualmente de diversas tecnologías que le permitan recolectar datos valiosos para favorecer la productividad, interpretando $\mathrm{y}$ aplicando información. Por consiguiente, la agricultura de precisión genera el ahorro de recursos disminuyendo el impacto ambiental así como costos.

Por otra parte, la disponibilidad y bajo costo de la informática móvil ha permitido incluir esta tecnología en la agricultura, como lo es un sistema de notificaciones diarias a los agricultores con información relacionada con la meteorología (Channe, Kothari y Kadam, 2015).

Romero y Amparo (2019) en su investigación, detallan la importancia de la implementación de programas pre-requisitos de análisis de peligros y puntos críticos de control (HACCP) en la Industria alimentaria. Su investigación se enfoca al desarrollo de un software que incorpora una base de datos relacional y genera un módulo de reportes para el control de capacitaciones al personal, control de limpieza, desinfección de los equipos, inspección de la planta y mantenimiento a equipos. Consideran que al automatizar el registro en los procesos, se reduce de manera significativa los tiempos optimizando actividades, generando además información digital que mejora la toma de decisiones.

Por otra parte, el uso de aguas regeneradas reducen la explotación de acuíferos y con un tratamiento adecuado permiten en la agricultura disminuir el consumo de fertilizantes. En base a lo anterior, la Unión Europea actualmente está analizando varias iniciativas para reutilizar aguas residuales urbanas e industriales, lo cual permitirá generar una real economía circular del agua (Melgarejo, 2019). 


\section{Objetivos}

\section{Objetivo general:}

Implementar una plataforma que integre aplicaciones de escritorio, Web y móviles, así como herramientas de Internet de las cosas, para el control eficiente en las huertas aguacateras.

\section{Objetivos específicos:}

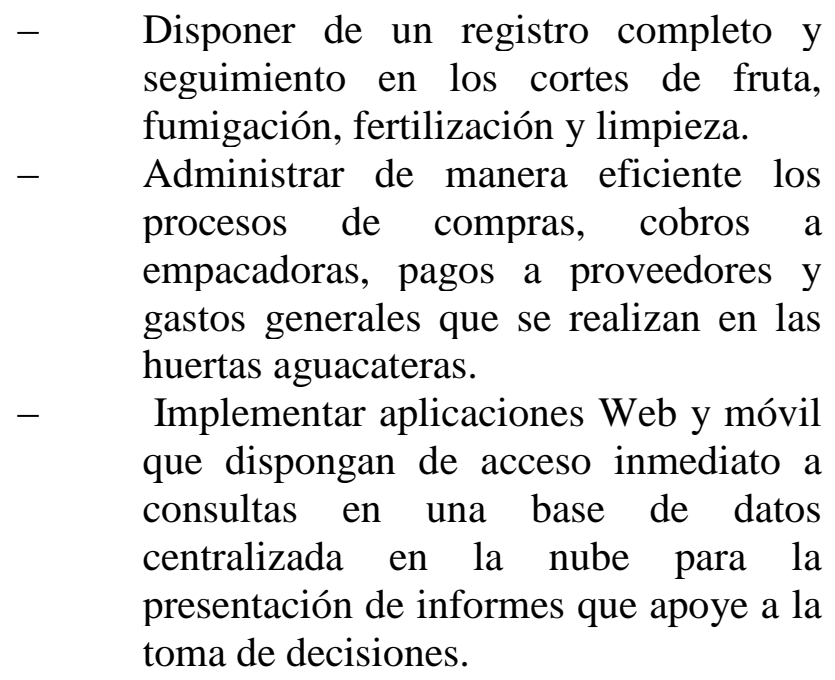

\section{Marco teórico}

\section{IoT}

Internet de las cosas o IoT (Internet of Things, por sus siglas en inglés), se refiere a conectar objetos tecnológicos o electrónicos a Internet para compartir y controlar las cosas que nos rodean, utilizando redes basadas en el protocolo IP, capacidad de cómputo, miniaturización, análisis de datos y computación en la nube. Algunas de sus aplicaciones son en las áreas de salud, herramientas de aprendizaje, seguridad, optimización de procesos y agricultura entre otros (Bautista, Parra-Valencia y Guerrero, 2017).

Las recientes innovaciones de la electrónica y las Tecnologías de la Información y Comunicación permiten un crecimiento en el procesamiento de los sistemas de tratamiento de información y a la vez han generado la miniaturización de microprocesadores empleados como son los sensores para captar datos del entorno. Así también, la expansión de Internet y la gestación de nuevas tecnologías, servicios y plataformas han generado el IoT como la próxima revolución de un mundo interconectado (Barrio, 2018).
El IoT implica el uso de RFID (Radio Frequency Identification, por sus siglas en inglés), así como red inalámbrica y sensores que incorporan una pila de Internet al dispositivo. Beagle black es un dispositivo IoT económico que se conecta a sensores para recoger información de las propiedades del suelo y de parámetros atmosféricos, los datos son recopilados y enviados a un almacenamiento en la nube para posteriormente ser procesados y de esta manera predecir el rendimiento del cultivo. Sin embargo, en la agricultura, pocos investigadores han propuesto arquitecturas basadas en IoT. (Channe, Kothari y Kadam, 2015).

En el sector primario, específicamente en la agricultura, es posible aprovechar el potencial de IoT, sin embargo, también genera amenazas referentes a la intimidad-privacidad y a la seguridad. El IoT ha sido llamado como un agente de la Industria 4.0, junto con la inteligencia artificial, la robótica, la impresión 3D y $4 \mathrm{D}$, la nanotecnología y la biotecnología, entre otros. Internet intercomunica ordenadores con diversos dispositivos (teléfonos inteligentes, tabletas, pulseras inteligentes, gafas de realidad aumentada, cámaras de seguridad, controles de acceso, sensores de temperatura, entre otros) para enviar datos a centros de procesamiento, transmitiendo, compilando y analizando datos. El IoT crece diariamente y se espera un número de veinte billones de dispositivos conectados para el año 2020 (Barrio, 2018).

\section{Computación Móvil}

Ramírez, Contreras y Contreras (2014) definen a un dispositivo móvil como un aparato diseñado para funciones específicas, de pequeño tamaño, con capacidades de procesamiento, conexión permanente o intermitente a una red y memoria limitada. Existen varios tipos de dispositivos móviles, desde los reproductores de audio portátiles hasta los navegadores GPS, incluyendo los teléfonos móviles, PDA's o las tabletas. En la programación de móviles existen plataformas que incluye herramientas como el SDK (Software Development Kit, por sus siglas en inglés), el IDE (Integrated Development Environment, por sus siglas en inglés) y emuladores, en algunos casos también se incluyen características como el GPS y acelerómetro, entre otros. 


\section{Minería de datos}

Consiste en un proceso de análisis de datos para localizar patrones ocultos. Se ha aplicado en la agricultura para el análisis y clasificación de los tipos y propiedades del suelo, predecir cultivos, así como decidir la mejor secuencia de cultivo basado en información de secuencias anteriores que incluyen un detalle de los nutrientes del suelo. Las propiedades del suelo como nitrato, fosfato y potasio determinan la calidad del suelo, así como el tipo de producción de los cultivos. Las propiedades ayudan a mantener la salud del suelo aplicando la cantidad necesaria de fertilizantes (Channe, Kothari y Kadam, 2015).

\section{Big Data}

Trejo (2018) considera que el concepto Big Data se refiere a grandes volúmenes de datos tanto disponibles como generados en tiempo real, que pueden ser alimentados desde múltiples orígenes ya sean locales o desde la nube, algunos extraídos de los usuarios y provenientes de distintos formatos, pero con el único objetivo de ser utilizados para el análisis descriptivo, predictivo o prescriptivo. El IoT se considera como una importante contribución de datos para el Big Data, ya que a través de una conexión de red transfiere datos que se encuentren relacionados con máquinas o dispositivos promoviendo la inteligencia artificial, así como el aprendizaje automático.

De esta manera, Big Data representa una cantidad masiva de datos captadas por sensores, redes sociales y sistemas comerciales que permite la captura, almacenamiento, análisis y búsqueda para gestionar la cadena de suministro de productos agrícolas disminuyendo costos de producción (Channe, Kothari y Kadam, 2015).

\section{Mecatrónica}

Kriuchkova, Canteñs, y Cabreray (2019) consideran a la mecatrónica como una tecnología con un enfoque multidisciplinario de la ingeniería que permite mantener altos estándares de competitividad en países industrializados y puede ser aplicada para dar solución a problemas relacionados con la agricultura, ya que involucra varias áreas de conocimientos en sistemas mecánicos, diseño de software, así como automatización y control.
Algunos proyectos basados en mecatrónica es un sistema de control de posición del disco de corte de una deshojadora de mazorcas con visión artificial que permite disminuir el error en el corte por el usuario por medio del empleo de controladores PID mediante una interfaz gráfica en MATLAB. Otro trabajo relevante es un robot de palmeras que considera una estructura con un mecanismo de biela-manivela para ascender, que incluye motores, batería y cámara IP para obtener imágenes y procesarlas por medio de visión artificial detectando cocos y enfermedades en las hojas. Otras técnicas de inteligencia artificial en modelado y control han sido utilizadas en los invernaderos para mejorar la producción. (Kriuchkova, Canteñs, y Cabreray (2019).

\section{Cloud Computing}

La computación en la nube tiene su aplicación en el sector agrícola ya que provee servicios rentables en el almacenamiento de información histórica importante para la aplicación de fertilizantes por medio de imágenes de cultivo que son recopiladas por drones, así como datos del ambiente alimentados por medio de sensores. De esta manera, los datos almacenados permiten construir un modelo, monitorear situaciones adversas y atenderlas oportunamente (Channe, Kothari y Kadam, 2015).

\section{Visual Studio}

Visual Studio desarrollado por Microsoft, ofrece una interfaz de desarrollo integrado con diversas herramientas para crear aplicaciones en plataformas .NET. Visual Studio se distribuye en diferentes ediciones como son Community, Professional y Enterprise. En sus últimas versiones se ha disminuido el tiempo de carga controlando además el rendimiento de las extensiones. Además, las aplicaciones pueden especificar las versiones del Framework .NET que se deben utilizar. Una de las herramientas que integra es un editor de texto el cual evalúa los errores de sintaxis y variables declaradas que no usan código. También incluye IntelliSence que es una funcionalidad para mostrar clases y sus miembros del código que se va escribiendo. Además, considera un diseñador de vistas de los elementos del proyecto, un depurador integrado para seguir la ejecución del código, así como un administrador de extensiones y actualizaciones (Hugon, 2018). 
En la versión 2019 de Visual Studio se incluyen mejoras significativas en el rendimiento IntelliSence para los archivos $\mathrm{C}++$, ofreciendo el desarrollo local con emuladores diversos, acceso simplificado a pruebas, así como la creación de repositorios y administración GIT en IDE. Ofrece además conjuntos de herramientas y componentes como Web \& Cloud para desarrollo ASP.NET y desarrollo Web, Azure, Python y Node.js, desarrollo móvil con .NET, desarrollo multiplataforma de .NET Core, almacenamiento y procesamiento de datos y el desarrollo de aplicaciones de analítica y de ciencia de datos (Microsoft, 2019).

\section{Android Studio}

Android surge desde una empresa emergente que desarrollaba este sistema operativo para cámaras digitales, fue adquirido por Google en 2005. La primera versión para dispositivos móviles aparece en 2008 y aproximadamente 40 versiones se han publicado posteriormente. Cada versión se caracteriza por el nivel de API (API Level en inglés). Este nivel de API es utilizado para distinguir la versión de Android instalada para el desarrollo de aplicaciones. En este sentido, Google provee, cada quince días, estadísticas relacionadas con las tasas de instalación de cada versión.

El sistema operativo de Android está basado en un núcleo Linux, el cual gestiona las capas básicas de los procesos, memoria y hardware, así como los permisos de los usuarios. La segunda capa presenta las bibliotecas principales del sistema como lo es la gestión de la visualización, motor de base de datos, gestión de audio y video entre otros. Las bibliotecas se utilizan directamente por los programadores para crear las aplicaciones móviles (HÉBUTERNE, 2018).

\section{Metodología}

Para el desarrollo de la plataforma que comprende aplicaciones de escritorio, Web y móviles, se aplicó la metodología de Scrum que es un marco de programación ágil que se basa en una estrategia de desarrollo iterativo e incremental que asegura la calidad del producto. A continuación, se detallan las diferentes fases de desarrollo.

\section{Análisis}

Se realizó una investigación de los requerimientos para el control de producción y comercialización en las huertas aguacateras llevando a cabo una serie de entrevistas.

La solicitud de los usuarios en cuanto a informes y consultas fue la siguiente:

- Relación de existencias de productos.

- Informe de actividades por periodo, huerta, tipo de actividad y responsable.

- Cobros pendientes por periodo, huerta o empacadora.

Cobros realizados por periodo, huerta o empacadora.

Compras en un periodo por proveedor.

- Compras pendientes para pagar.

- Cortes en un periodo por empacadora o huerta.

- $\quad$ Pagos en un periodo por proveedor.

- $\quad$ Gastos en un periodo por concepto.

En la Figura 1 se presenta el diagrama de casos de uso de la plataforma informática diseñada.

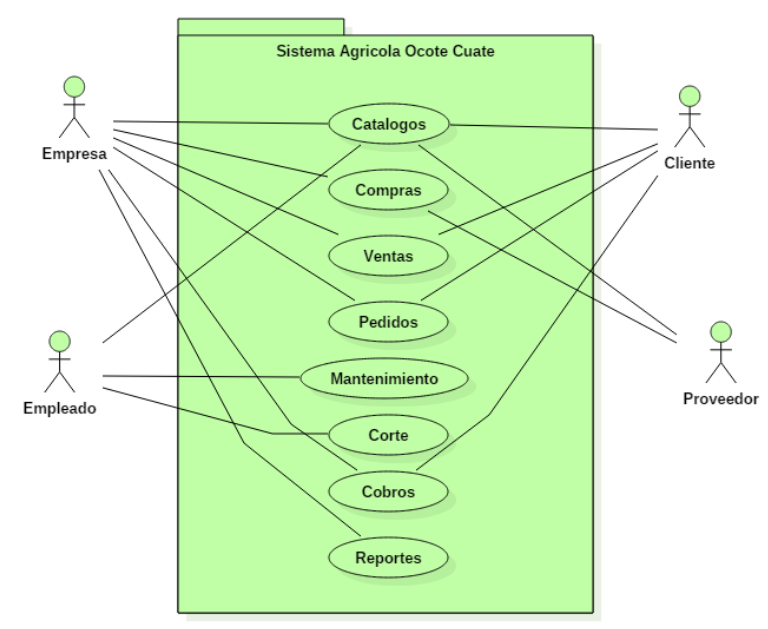

Figura 1 Diagrama de casos de uso general

Un requerimiento importante para los encargados de las huertas fue el registro y seguimiento a las actividades en el campo, ya que se debe tener especial cuidado en la aplicación de agroquímicos, mantenimiento y seguimiento en tiempos para efectuar la tarea de manera apropiada y oportuna, sin causar daños al cultivo o a la producción, evitando además costos innecesarios.

Algunas de las actividades que se realizan en las huertas de aguacate se muestran a continuación: 


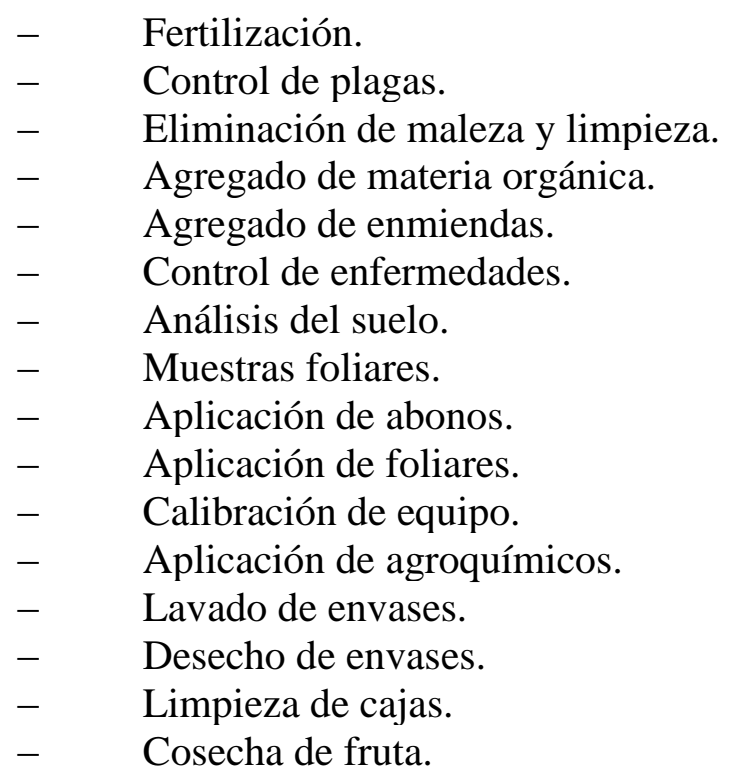

Otras de las consideraciones al capturar los requerimientos del sistema fue que los productos que se aplican en las huertas, así como el equipo y maquinaria utilizado deben estar de manera permanente inventariados. Por lo anterior, fue necesario considerar actualizar la existencia de los productos cada vez que eran aplicados durante una actividad o bien adquiridos mediante una compra.

En relación con los cortes, las empacadoras se presentan en las huertas para llevar a cabo directamente esta actividad bajo una alta supervisión. La fruta es transportada y pesada en cajas plásticas. Los datos generales del corte deben quedar correctamente registrados, ya que representa un adeudo que la empacadora deberá pagar posteriormente al productor de la huerta. Estos movimientos deberán quedar registrados en la plataforma para evitar contratiempos durante la cobranza. De esta manera, también se pueden rastrear otros datos importantes sobre los cortes. Otros requerimientos solicitados incluyen el control sobre los gastos diversos que las huertas generan, por lo cual, se debe tener un catálogo de conceptos autorizados. En relación a las compras a proveedores pueden ser a crédito o contado, siendo necesario llevar un seguimiento detallado del saldo del proveedor.

\section{Diseño}

Se diseñó una base de datos compartida para los tres tipos de escenarios (escritorio, Web y móvil), la cual fue creada en MySQL y almacenada en un servidor en la nube. La base de datos estuvo integrada por las siguientes entidades:

$\begin{array}{ll}- & \text { Usuarios } \\ - & \text { Empacadoras } \\ - & \text { Proveedores } \\ - & \text { Productos } \\ - & \text { Encargados } \\ - & \text { Huertas } \\ - & \text { Tipo de Cobro-Pago } \\ - & \text { Concepto de gastos } \\ - & \text { Tipos de productos } \\ - & \text { Tipo Actividad } \\ - & \text { Actividades } \\ - & \text { Detalle Actividades } \\ - & \text { Compras } \\ - & \text { Detalle Compra } \\ - & \text { Cortes } \\ - & \text { Cobros } \\ - & \text { Gastos } \\ - & \text { Pagos }\end{array}$

Los procesos que intervienen en las actividades diarias que realizan las huertas se mencionan a continuación:

- $\quad$ Registro y consulta de actividades.

- $\quad$ Registro y consulta de compras a crédito y contado.

- $\quad$ Registro y consulta de cortes.

- Registro y consulta de cobros.

- Registro y consulta de gastos.

- $\quad$ Registro y consulta de pagos.

De igual forma, se realizó el maquetado de las interfaces en cada tipo de aplicación, así como el diseño de los informes utilizando ReportViewer en la aplicación de escritorio y la librería fpdf para generar informes PDF en la aplicación Web.

$\mathrm{Al}$ incluir un apartado de utilerías dentro de las opciones de la plataforma, fue necesario diseñar la lógica para el respaldo y la restauración de la base de datos desde MySQL, control de bitácora de usuarios, ayuda en línea y traspaso de movimientos a una base de datos histórica.

\section{Codificación}

Se detalló una estructura de opciones y componentes para cada aplicación, tomando en consideración el perfil del usuario. También se emplearon diferentes herramientas y lenguajes de programación, entre ellos Visual Studio, HTML, Bootstrap, PHP, JavaScript, Ajax, Java y Android Studio. 
Al desarrollar las aplicaciones fue indispensable realizar pruebas de conexión a la base de datos en MySQL instalando controladores e incorporando las clases correspondientes en la lógica de las aplicaciones de cada escenario.

\section{Pruebas}

Para verificar la funcionalidad de la plataforma fue necesario realizar pruebas individuales y de concurrencia en cada caso de uso, por lo cual, se diseñaron casos y procedimientos de prueba. Una vez asegurada la funcionalidad de cada componente, fue solicitada la revisión y autorización del avance del proyecto de parte de los usuarios de la plataforma, donde se realizaron algunos ajustes que se consideraban necesarios.

Posteriormente, al concluir el desarrollo completo correspondiente a un escenario específico, se realizaba una prueba integradora con la finalidad de descubrir fallas y corregir los defectos de manera oportuna antes de liberar la aplicación. Esta revisión permitió asegurar y certificar la entrega final del proyecto. En la Tabla 1 se muestra el caso de prueba para el movimiento de cortes donde se describen los valores de entrada, las condiciones establecidas al momento de realizar la prueba, así como la respuesta a la salida que debe generar el sistema.

\begin{tabular}{|c|c|}
\hline $\begin{array}{l}\text { Nombre del caso } \\
\text { de uso }\end{array}$ & Registrar Corte \\
\hline Descripción & $\begin{array}{l}\text { Registrar un corte en el sistema con } 250 \\
\text { cajas, } 500 \text { toneladas y } \$ 700,000 \text { de } \\
\text { importe. }\end{array}$ \\
\hline Datos de entrada & $\begin{array}{l}\text { idCorte: } 1 \\
\text { Huerta: El Cincuenta } \\
\text { Empacadora: Los cerritos } \\
\text { Número de Cajas: } 250 \\
\text { Fecha: } 12 \text { de abril del } 2019 \\
\text { Toneladas: } 500 \\
\text { Ingreso Generado } \$ 700,000\end{array}$ \\
\hline Condiciones & $\begin{array}{l}\text { El saldo inicial de la empacadora es } \$ 500 \text {. } \\
\text { El número de cajas es } 250 \text {, las toneladas } \\
500 \text { y el importe de } \$ 700,000 \text {. }\end{array}$ \\
\hline $\begin{array}{l}\text { Secuencia } \\
\text { Principal }\end{array}$ & $\begin{array}{ll}\text { 1. } & \text { El usuario presiona Nuevo para } \\
\text { generar el IdCorte. } \\
\text { 2. }\end{array}$ \\
\hline Salida & $\begin{array}{l}\text { Graba nuevo registro en cortes } \\
\text { Saldo Empacadora }=\$ 700,500\end{array}$ \\
\hline
\end{tabular}

Tabla 1 Prueba registrar corte

\section{Documentación}

Durante el desarrollo de la plataforma se diseñó el manual de programación que permitirá dar seguimiento al proyecto. Este manual consiste en una guía de fácil acceso a todo el análisis y diseño de la plataforma, que incluye información sobre diagramas de despliegue, clases, diccionario de datos, casos de uso, actividad, estado y colaboración, así como una descripción detallada de los componentes de programación generados durante la implementación del sistema. Por otra parte, se diseñó una ayuda en línea, la cual es un apoyo de consulta para los usuarios sobre la operación del sistema.

\section{Resultados}

En este apartado se presentan algunas de las aplicaciones generadas a través de la plataforma. La Figura 2 muestra la interfaz para el registro de huertas donde se captura responsable, nombre de huerta y ubicación.

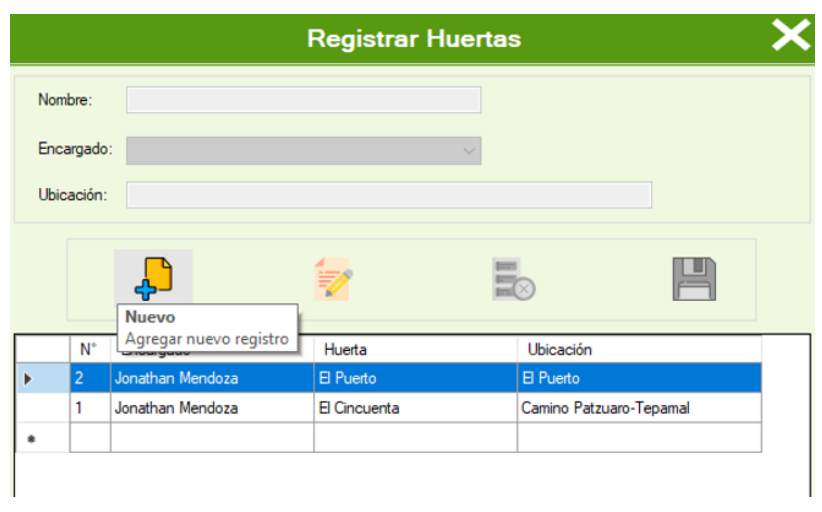

Figura 2 Registro de huertas

En la Figura 3 se muestra la interfaz para registrar los cortes que realiza cada una de las empacadoras en las huertas. Se selecciona la huerta y la empacadora, fecha de corte y número de cajas. Posteriormente, se capturan las toneladas y el importe. También se despliega una consulta general de los cortes de la huerta actualmente registrados.

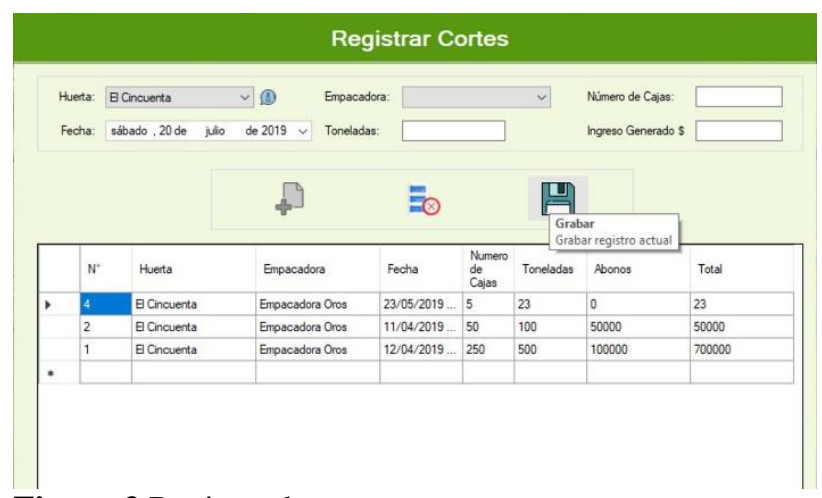

Figura 3 Registro de cortes

OCHOA-ORNELAS, Raquel, FAJARDO-DELGADO, Daniel, SÁNCHEZ-CERVANTES, María Guadalupe y OSORNIO-MENDOZA, Jonathan. Implementación de Aplicaciones Informáticas en la Industria Agrícola del Aguacate. Revista del Desarrollo Tecnológico. 2019 
En la Figura 4 se presenta la interfaz del registro de cobros a empacadoras, la cual integra una consulta general de cortes pendientes a cobrar. Una vez que se elige el corte a cobrar, se muestra en detalle los datos correspondientes para introducir el importe, tipo de cobro y fecha.

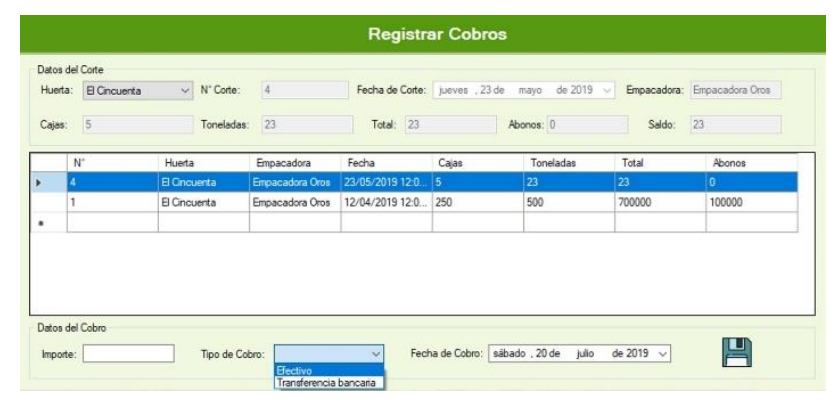

Figura 4 Registro de cobros

Algunos de los reportes que se generan en la aplicación Web es la relación de empacadoras con saldo pendiente, así como el reporte de cortes, ambos en un periodo previamente seleccionado por el usuario, ver Figuras 5 y 6.

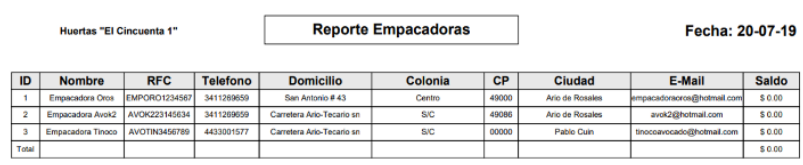

Figura 5 Reporte de empacadoras

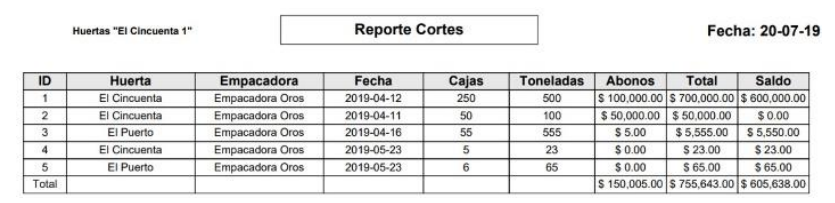

Figura 6 Reporte de cortes

En la Figura 7 se presenta la interfaz para acceder a los catálogos desde el dispositivo móvil.

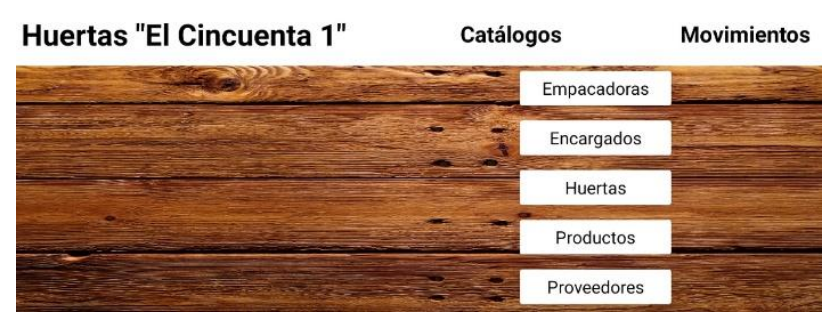

Figura 7 Catálogos aplicación móvil

En la Figura 8 se presenta la consulta de los datos de una empacadora desde el dispositivo móvil.

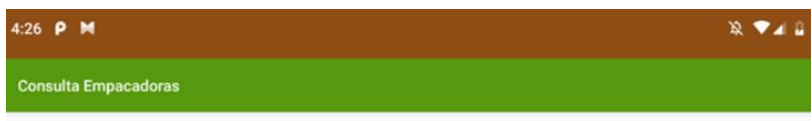

Nombre...Empacadora Tinoco RFC...AVOTIN3456789 Teléfono...4433001577 Domicilio...Carretera Ario-Tecario sn Colonia...S/C CP...00000 Ciudad...Pablo Cuin eMail....tinocoavocado@hotmail.com Saldo...\$0

Figura 8 Consulta individual de empacadora

\section{Conclusiones}

Al implementar las nuevas tecnologías en las huertas aguacateras, se puede obtener mayor confiabilidad en las tareas diarias que se realizan durante el cultivo, cosecha, administración y procesos de comercialización. Además, la plataforma brinda información de manera oportuna generando una mejora y crecimiento a las unidades productivas.

El seguimiento de los registros en todo el proceso productivo del aguacate, se pueden identificar puntos críticos en caso de presentarse una emergencia sanitaria y retirar los productos contaminados sin perjudicar al resto de la producción. También permite disponer de información relacionada con las tareas concernientes a las aplicaciones de fertilizantes, plaguicidas, dosis, fechas, problema a atender y responsables. Además, es posible obtener un listado de existencia de insumos, fechas de ingreso y salida, consultas de cosechas con responsables de mantenimiento y limpieza, fechas de entrega de la fruta, cantidad entregada y empacadora. Considerando lo anterior, se genera una eficiente trazabilidad de las actividades operativas que están involucradas en todo el proceso.

Para tener acceso a los sistemas informáticos desde cualquier escenario, es indispensable disponer de una eficiente cobertura a Internet, lo cual fue posible en este caso para comprobar el comportamiento de las aplicaciones y realizar las pruebas necesarias, contribuyendo a apoyar los procesos administrativos que se realizan en las huertas aguacateras.

La ingeniería de software permite extraer conocimiento de las actividades que se llevan a cabo en el campo, evitando pérdida de información y manteniendo un mejor control y trazabilidad, mejorando utilidades y reduciendo riesgos a la pérdida de capitales o destrucción de recursos naturales, ya que ayuda a mantener un control de las huertas a distancia por medio de las aplicaciones para dispositivos móviles.

OCHOA-ORNELAS, Raquel, FAJARDO-DELGADO, Daniel, SÁNCHEZ-CERVANTES, María Guadalupe y OSORNIO-MENDOZA, Jonathan. Implementación de Aplicaciones Informáticas en la Industria Agrícola del Aguacate. Revista del Desarrollo Tecnológico. 2019 
Es posible reducir la brecha digital entre la resistencia de los agricultores al uso de los medios informáticos identificando los beneficios que la informática garantiza implementando además programas de formación al uso de la tecnología.

La plataforma desarrollada actualmente se encuentra en una fase de implantación y capacitación a usuarios. Por otra parte, este proyecto cubre el control administrativo de las aguacateras y puede ser adaptado a otro tipo de huertas. Adicionalmente se continuará con el desarrollo de software para integrar el análisis de datos en relación a los suelos en los cultivos por medio de un registro de datos histórico de los nutrientes.

Con el desarrollo del proyecto, se obtuvo experiencia en la implementación de la tecnología en la agricultura, misma que beneficia a los estudiantes de Ingeniería Informática en la línea de investigación "Ingeniería de software e infraestructura" con clave LGAC-2017-CDGU-IINF-19, fortaleciendo además el cuerpo académico "Algoritmos y optimización / Visión computacional, sistemas concurrentes y tecnologías de software" con clave ITCGUZCA-7.

\section{Referencias}

Barrio, M. (2018). Internet de las cosas, Madrid, España: REUS

Bautista, D. R., Parra-Valencia, J. A., \& Guerrero, C. D. (2017). IOT: Una aproximación desde ciudad inteligente a universidad inteligente. Revista Ingenio Universidad Francisco de Paula Santander Ocaña, 13(1), 920.

Bernal, F. C., Fernández, E. M., \& Aparicio, G. G. (2019). Enfoque epistemológico del seguro agrario para la agricultura familiar en Bolivia. Apthapi, 5(1), 1517-1523.

Calvo, A. C. (2019). 1259. Sector privado y sector público en la industria española de alta tecnología: Indra. Biblio3W Revista Bibliográfica de Geografía y Ciencias Sociales, (1).
Channe, H., Kothari, S., \& Kadam, D. (2015). Multidisciplinary model for smart agriculture using internet-of-things (IoT), sensors, cloudcomputing, mobile-computing \& big-data analysis. Int. J. Computer Technology \& Applications, 6(3), 374-382.

Faostat, F. A. O., \& Production, A. C. (2016). Food and agriculture organization of the united nations, 2010. Roma, Italy.

HÉRBUTERNE， S. (2018). Desarrolle una aplicación Android / Programación en Java con Android Studio. Barcelona, España: Ediciones ENI.

Hugon, J. (2018). Desarrolle aplicaciones Windows con Visual Studio 2017. Barcelona, España: Editions ENI.

Kriuchkova, E. R., Canteñs, G. L., \& Cabrera, J. R. S. (2019). Mecatrónica en la universidad agrícola. Justificación de la especialidad en la Universidad Autónoma Chapingo, México. Revista internacional de aprendizaje en ciencia, matemáticas y tecnología, 6(1), 9-19.

Melgarejo, J. (2019). Agua y economía circular.

Méndez-Moreno, M., Quetz-Aguirre, G. M., Chuc-Armendáriz, M. B., Can-Cabrera, A. F., \& González-Uscanga, I. U. Códigos de Respuesta Rápida para la Optimización de Lectura en la Industria Agrícola y Floral: Caso Teya. AGROECOSISTEMAS TROPICALES, 34.

Microsoft (2019). Visual Studio 2019. Recuperado el 8 de Agosto de 2019 de https://visualstudio.microsoft.com/es/vs/

Ramírez, E. Contreras, O. y Contreras, C. (2014) . Programación móvil, recuperado el 31 de Julio de 2018 de http://programacionmovilufps.blogspot.com/20 14/11/que-es-la-programacion-laprogramacion.html

Perez-Garcia, C. A. (2019). Sistema de Información Geográfica para la agricultura cañera en la provincia de Villa Clara. Revista Cubana de Ciencias Informáticas, 13(2), 3046.a 
Pérez, M. P., \& Reyes, J. C. (2019). La concentración y centralización del capital en la agricultura latinoamericana. Implicaciones para el campesinado/The Concentration and Centralization of Capital in Latin

Romero, C., \& Amparo, R. (2019). Implementación de programas pre-requisitos de análisis de peligros y puntos críticos de control (HACCP) en la industria alimentaria a través de un software.

Sater, W. F. (2019). La agricultura chilena y la Guerra del Pacífico. revista historia, (16), 125149 .

Trejo, D. (2018). Big data, una oportunidad de mejora en las organizaciones. México: DSA IyDA A.C.

Zarazaga F.J. (2017). Agricultura 4.0: Las tecnologías de la industria 4.0 aplicadas al campo. Recuperado el 8 de Agosto de 2019 de https://www.geoslab.com/es/blog/agricultura40-las-tecnologias-de-la-industria-40-aplicadasal-campo 